\title{
Abstracts of the 17th European Workshop on Neonatology
}

Vila do Conde, Portugal, September 23-26, 2009

Supported by VYGON Portugal, Lda

Effectiveness of skin conductance in assessing the nociceptive response from heel prick in neonates compared with the neonatal infant pain scale

L. Pereira-da-Silva ${ }^{\mathrm{a}}$, I. Monteiro ${ }^{\mathrm{a}}$, S. Gomes ${ }^{\mathrm{a}}$, P. Rodrigues $^{\mathrm{a}}$, D. Virella ${ }^{\mathrm{a}}$, M. Serelha ${ }^{\mathrm{a}}$ and H. Storm ${ }^{\mathrm{b}}$

${ }^{a}$ NICU, Department of Pediatrics, Hospital Dona Estefânia, Centro Hospitalar de Lisboa Central, Lisbon, Portugal

${ }^{\mathrm{b}}$ The Skills Simulation Center, Medical Faculty, University of Oslo, Norway

Background: Skin conductance (SC) is a recent noninvasive validated method and the Neonatal Infant Pain Scale (NIPS) is one of the most used clinical tools for assessing pain in neonates. While NIPS is a point in time assessment dependent on the observer, SC provides continuous quantitative measurements for as long as wished.

Aim: Effectiveness assessment of the SC index peaks per sec, compared with the NIPS for the response to heel prick in neonates.

Methods: Observational study of a systematic, convenience sample of neonates in a tertiary NICU with clinical indication for collecting blood by heel prick (blood gas or glycaemia). NIPS and SC index (MedStorm Stress Detector ${ }^{\mathrm{TM}}$ ) were simultaneously performed before, during and after the heel prick procedure, by two independent observers.

Results: 68 heel prick procedures (46 for glycaemia and 22 for blood gas analysis) were inflicted to 15 neonates. Both NIPS and SC index were significantly higher in the 30 seconds immediately after the stimulus than before or thereafter (Wilcoxon Signed Ranks Test; $p<0.001)$. Correlation of NIPS with the peaks per second that was achieved for blood gas sampling was better than for glycaemia $(r=0.554, p=0.008 \mathrm{vs}$. $r=0.165, p=0.279$ ).

Conclusions: Both NIPS and the SC index increased during heel stick. The correspondence between NIPS and the SC index varied with the time span of assessment. The longer the period of assessment, as in blood gas sampling, the better the correspondence between NIPS and SC.

Pressure drop during mechanial ventilation across small endotracheal tubes

R. Hentschel ${ }^{\mathrm{a}}$, J. Heukäufer ${ }^{\mathrm{b}}$, J. Guttmann ${ }^{\mathrm{b}}$ and S. Schumann ${ }^{\mathrm{b}}$

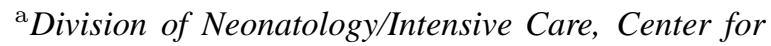
Pediatrics and Adolescent Medicine, University Hospital of Freiburg, Germany

${ }^{\mathrm{b}}$ Section for Experimental Anesthesiology, University Hospital of Freiburg, Germany

Purpose: Pressure drop (PD) across the endotracheal tube (ET) increases with decreasing diameter and, except for $2.0 \mathrm{~mm}$ ETs, can be calculated from published tube coefficients. Knowledge of PD may help to better fine-tune ventilation parameters and also gives an important measure of work of breathing during spontaneous breaths.

Methods: PD was measured in a model study with 2 model lungs $(\mathrm{C}=0.63$ or $0.40 \mathrm{ml} / \mathrm{mbar})$ during ventilation with a Babylog 8000 ventilator. While frequency was held constant at $40 / \mathrm{min}$ other ventilation parameters were varied within the normal range of conventional ventilation of preterms. All potential combinations of: peak pressure (PIP) 25/30/35/40 mbar, PEEP 4/6 mbar and inspiratory time (ti) 0.3/0.4/0.5/0.6 sec were studied. Using piezoelectric pressure transducers at the 
tube connector and at the tip of the ET 10 ventilation cycles of $30 \mathrm{sec}$ each were recorded for each ventilator setting at a sample rate of $250 \mathrm{~Hz}$. Mean difference of both pressures was analyzed using the root-meansquare error (rms-e).

Results: Important variables for PD were (in descending order): high PIP (maximum rms-e 7.7mbar), short ti (maximum rms-e 4.3mbar), low PEEP and high compliance. Mean rms-e for all pooled differences was $4.3+/-1.7 \mathrm{mbar}$

Conclusion: PD across a $2.0 \mathrm{~mm}$ ET is substantial. Using a short ti with a low PEEP and a high PIP will result in erroneously low tracheal pressure swings.

\section{Establishing enteral feeding (EF) in very low birth weight (VLBW) infants}

L.M.E. Ytrehus ${ }^{\mathrm{b}}$, M.H. Rostad ${ }^{\mathrm{b}}$ and D. Bratlid ${ }^{\mathrm{a}, \mathrm{b}}$

${ }^{a}$ Department of Pediatrics, St. Olavs University Hospital, Trondheim, Norway

${ }^{\mathrm{b}}$ Department of Laboratory Medicine, Children's and Women's Health, Faculty of Medicine, Norwegian University of Science and Technology, Trondheim, Norway

Introduction: Successful EF is a major treatment goal of VLBW infants. Few cohort studies have, however, been performed on this process.

Methods: Development of EF was analyzed retrospectively in a cohort of $46 \mathrm{VLBW}$ infants (gestational age (GA) $28.7 \pm 2.4$ weeks, birth weight (BW) 1139 \pm 254 gram) who survived to discharge.

Results: At regained BW (11.6 \pm 3.4 days) $67 \%$ of infants were on complete EF, $2 \%$ on total parenteral nutrition (TPN) and 30\% on mixed EF/TPN. Time to full EF was more related to postnatal age (PA) than to GA and was delayed in infants on long term antibiotics. At 34 weeks GA $93 \%$ of infants were only on EF. Of these, $44 \%$ were gavage fed (GF). Time to termination of GF was more related to $\mathrm{GA}(37.3 \pm 1.7$ weeks $)$ than to PA $(58 \pm 20$ days $)$, and was delayed in infants on long term CPAP. At discharge $57 \%$ of infants were exclusively breast fed. Infants with medical complications were less likely to be fully breast fed.

Conclusions: Most VLBW infants are breastfed at discharge. Maturation of gut physiology is more related to PA than GA. Maturation of sucking and swallowing is more related to GA than PA. Long term CPAP and complications delays this maturation.

The role of glycoprotein 130 (GP130) family of cytokines on fetal lung development mechanisms

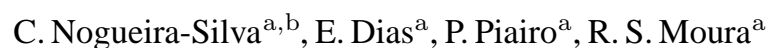
and J. Correia-Pinto ${ }^{\text {a,c }}$

${ }^{\mathrm{a}}$ Life and Health Sciences Research Institute (ICVS), School of Health Sciences, University of Minho, Braga, Portugal

${ }^{\mathrm{b}}$ Department of Gynecology and Obstetrics, Hospital S. Marcos, Braga, Portugal

${ }^{\mathrm{c}}$ Division of Pediatric Surgery, Department of Pediatrics, Hospital S. João, Porto, Portugal

Interleukin 6 (IL6), IL11, leukaemia inhibitory factor (LIF) and cardiotrophin-like cytokine (CLC) belong to the family of glycoprotein 130 (gp130)-type cytokines. These cytokines are aggregated in same family since all they use gp130 as signal transducer. Recently, it was demonstrated that IL6 is expressed in fetal pulmonary epithelium and enhances lung branching. Thus, our aim was clarify the role of IL11, LIF and CLC during fetal lung development.

Fetal rat lungs were harvested at 13.5 days postconception and cultured during 4 days with increasing doses of IL11 $(0,0.01,0.1,1,10,100 \mathrm{ng} / \mathrm{mL}), \operatorname{LIF}(0$, $0.4,4,20,40 \mathrm{ng} / \mathrm{mL})$ and CLC (0, 0.0003, 0.003, 0.03, $0.3,3,30 \mathrm{nM})$. LIF inhibition studies were also performed using neutralizing antibodies. Daily pictures were made and the total number of peripheral airway buds and epithelial perimeter were determined in lung explants. The results were expressed as Day 4/Day 0 ratio.

A total of 226 fetal lungs were dissected. IL11 supplementation induced no significant effect on lung growth, whereas LIF and CLC supplementation significantly decreased, in a dose-dependent way, the branching and epithelial perimeter. Additionally, the inhibition of LIF action increased the measured parameters.

Although these cytokines share a common signal transducer, these findings suggest specific biological activities on lung development. This specificity can represent a regulatory mechanism of lung morphogenesis, intrinsic to this family, in order to achieve the correct lung growth.

Incidence of early onset neonatal sepsis (EONS) in the era of antenatal antibiotics (AA): A regional population based study

P. Kuhn and J. Messer Neonatology, University Hospital Strasbourg, France

In 2001, recommendations for use of AA have been implemented in France: Intrapartum Antimicrobial 
Phophylaxis (IAP) to prevent group B streptococcal (GBS) disease and AA to prolong pregnancy in case of Preterm Premature Rupture Of Membranes (PPROM).

Purpose: To determine the effects of AA on the incidence and ecology of EONS.

Methods: We designed a population based prospective observational study over 1 year (March 2004February 2005) in levels III and II centres troughout Alsace. All neonates identified for confirmed or probable EONS were included.

Results: 20131 neonates were born during the period. 164 were included: 24 had confirmed sepsis, 140 probable sepsis. The incidence of confirmed EONS was $1.19 / 1000$ births. The type of bacteria was GBS in $15 / 24$ cases $(62 \%)$ of confirmed EONS (incidence of $0.75 / 1000)$.

E. Coli was identified in $6 / 24(25 \%)$ confirmed EONS (incidence of 0.3/1000). Among E. Coli infections $(n=36)$, Amoxicillin resistance $(n=18)$ was statistically linked with AA use (13/18): $p=0.009$. This risk appeared greater in preterm infants born after AA for PPROM than in term infants born after IAP to prevent GBS infections.

In about $30 \%$ of cases, AA were not administered despite positive screening or presence of risk factors.

Conclusions: GBS remains the predominant pathogen. AR E. Coli infections seem to be linked to prolonged AA in case of PPROM and affect preferentially preterm infants. Postnatal treatment strategies should take into account this effect. The current policy of GBS prophylaxis does not appear to increase risk of resistant pathogens. Progress could result from better compliance to guidelines.

\section{A score predicting late-onset sepsis in VLBW infants}

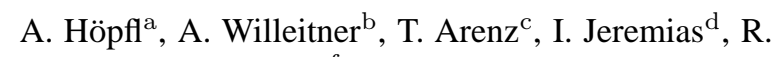
Geiger $^{\mathrm{e}}$ and H. Küster ${ }^{\mathrm{f}}$

${ }^{a}$ Children's Hospital Landshut, Landshut, Germany

${ }^{\mathrm{b}}$ Children's Hospital Oklahoma, Oklahoma, OK, USA

${ }^{\mathrm{c} C h i l d r e n ' s ~ H o s p i t a l ~ B e r n, ~ B e r n, ~ S w i t z e r l a n d ~}$

${ }^{\mathrm{d}}$ Helmholtz Center Munich, Munich, Germany

e Children's Hospital Innsbruck, Innsbruck, Austria

${ }^{\mathrm{f}}$ Children's Hospital Greifswald, Greifswald, Germany

Purpose: To establish a score to early predict lateonset sepsis specifically in very low birth weight infants.

Design: Two prospective multi-centre studies in very low birth weight infants were used: infants in the first served as calculation cohort, those in the second as validation cohort.

Methods: Infants were divided into a sepsis group, a non-sepsis group, and an undetermined group by predefined criteria. 39 parameters recorded daily were compared in the sepsis and the non-sepsis group between Day -3 and Day 0. The score was created combining those parameters with the highest area under the ROC curve.

Results: A score was generated consisting of the following five clinical and laboratory categories. When positivity for sepsis was defined as pathological findings in at least 2 out of these 5 categories, the score reached a sensitivity of $84.0 \%$ and a specificity of $91.7 \%$ in the validation cohort in predicting late onset sepsis.

Conclusion: We created a highly predictive score for early diagnosis of late-onset sepsis in very low birth weight infants. The score may be of value when comparing infants with sepsis in multi-center studies.

\section{Treatment characteristics and in-hospital treatment costs for very low birth weight (VLWB) infants in Norway}

A. Nordermoen ${ }^{\mathrm{b}}$ and D. Bratlid ${ }^{\mathrm{a}, \mathrm{b}}$

${ }^{a}$ Department of Pediatrics, St. Olavs University Hospital, Trondheim, Norway

${ }^{\mathrm{b}}$ Department of Laboratory Medicine, Children's and Women's Health, Faculty of Medicine, Norwegian University of Science and Technology, Trondheim, Norway

Introduction: Improved treatment has increased survival of VLBW infants. We have retrospectively compared treatment and in-hospital costs for VLBW infants born in 1997 and 2007.

Methods: Individual costs (diagnostics, medications, surgery) and treatment level (I, II or III) were analysed from patient records. Personnel costs were calculated from departmental and hospital data.

Results: In 2007 more infants were delivered by csection (84\% vs 65\%), received more TPN $77 \%$ vs $61 \%$ ), but not surfactant (56\% vs. 55\%). Survival was also higher (94\% vs. 83\%), also for infants with BW $<1000 \mathrm{~g}$ (85\% vs $75 \%)$. Survival of infants without BPD, ROP, IVH, PVL, NEC increased from $51 \%$ in 1997 to $69 \%$ in 2007, while time to death was reduced $(19 \pm 33$ vs $12 \pm 11$ days $)$. Total individual treatment costs were unchanged and counted for $<10 \%$ of total costs. Total treatment costs pr. patient increased 
from $€ 67.700$ in 1997 to $€ 75.300$ in 2007 , while costs pr. healthy survivor was reduced from $€ 130.100$ to $€ 108.500$. However, treatment costs for healthy survivors with $\mathrm{GA}<28$ weeks increased from $€ 349.000$ in 1997 to $€ 429.500$ in 2007.

Conclusions: Treatment costs of VLBW infants are strongly related to personnel costs. Premature infants with GA $<28$ weeks are probably hospitals most expensive patients.

\section{Bronchopulmonary dysplasia: Clinical practices in five Portuguese neonatal intensive care units}

H. Guimarães ${ }^{\mathrm{a}, \mathrm{g}}$, G. Rocha ${ }^{\mathrm{a}, \mathrm{g}}$, G. Vasconcellos ${ }^{\mathrm{a}}$, E. Proença $^{\mathrm{b}}$, M.L.Carreira ${ }^{\mathrm{c}}$, M.R. Sossai ${ }^{\mathrm{d}}$, B. Morais ${ }^{\mathrm{d}}$, I. Martins $^{\mathrm{e}}$, T. Rodrigues ${ }^{\mathrm{f}, \mathrm{g}}$ and M.Severo ${ }^{\mathrm{f}, \mathrm{g}}$

${ }^{a}$ Hospital de S. João, Porto, Portugal

${ }^{\mathrm{b}}$ Maternidade Júlio Dinis, Porto, Portugal

${ }^{\mathrm{c}}$ Hospital de Santo António, Porto, Portugal

${ }^{\mathrm{d}}$ Hospital Fernando Fonseca, Lisboa, Portugal

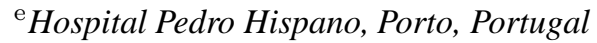

${ }^{\mathrm{f}}$ Department of Epidemiology, Faculty of Medicine of Porto University, Portugal

${ }^{\mathrm{g}}$ Faculty of Medicine of Porto University, Portugal

Aim: To compare five Portuguese NICUs, regarding the clinical practices on very low birth weight infants, in order to develop better practices to prevent bronchopulmonary dysplasia (BPD).

Patients and Methods: 256 preterm neonates, gestational age $(\mathrm{GA})<30$ weeks and/or birthweight $(\mathrm{BW})$ $<1250 \mathrm{~g}$ admitted at five Portuguese NICUs (centers 1 to 5) between 2004 and 2006, were studied. BPD was defined as oxygen dependency at 36 weeks of postconceptional age. We considered clinically significant a practice to be improved whether a decrease greater than $10 \%$ in the prevalence of BPD was achieved, using a statistical model adjusted for the practice, GA and BW.

Results: The overall prevalence of BPD was $12.9 \%$. Our results revealed that prenatal corticosteroids (PNC) should be improved in centers 2, 4 and 5; fluid policy should be improved in centers 3 and 5; oxygen therapy should be improved in centers 1 and 3; sepsis prevention should be improved in centers 1 and 2; and PDA treatment should be improved in center 2 .

Conclusion: The implementation of potentially better practices to reduce lung injury in neonates, in these 5 Portuguese NICUs, must be addressed to improve the policies on $\mathrm{PNC}, \mathrm{FiO}_{2}$, fluid ministration, sepsis and PDA treatment.
The role of angiotensin II during fetal lung development: A possible molecular explanation for fetal lung hypoplasia induced by inhibitors of reninangiotensin system

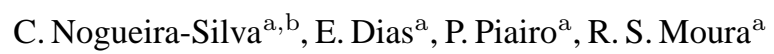
and J. Correia-Pinto ${ }^{\mathrm{a}, \mathrm{c}}$

${ }^{\mathrm{a}}$ Life and Health Sciences Research Institute, School of Health Sciences, University of Minho, Braga, Portugal ${ }^{\mathrm{b}}$ Department of Gynecology and Obstetrics, Hospital S. Marcos, Braga, Portugal

${ }^{\mathrm{c}}$ Division of Pediatric Surgery, Department of Pediatrics, Hospital S. João, Porto, Portugal

Background: Maternal administration of angiotensin converting enzyme inhibitors (ACE-I) and angiotensin receptor-antagonists (ARA) can induce fetal lung hypoplasia. However, the role of renin-angiotensin system (RAS) on lung morphogenesis remains unknown. Our aim was to clarify the role of angiotensin II (AngII) during fetal lung development.

Methods: Fetal rat lungs were harvested at 13.5 days post-conception and cultured with increasing doses of AngII or selective antagonists of AngII type $1\left(\mathrm{AT}_{1} \mathrm{R}\right.$; ZD-7155) and 2 ( $\mathrm{AT}_{2} \mathrm{R}$; PD-123319) receptors. Morphometric analysis was performed in all explants. Explants treated with AngII, ZD-7155 and PD-123319 were assessed by Western blot for STAT3, ERK1/2, JNK, p38, PI3-AKT phosphorylation.

Results: AngII supplementation induced a biphasic effect (lower doses increased, while higher doses did not accomplish additional effect) on lung growth. Selective activation of $\mathrm{AT}_{1} \mathrm{R}$ (using $\mathrm{AT}_{2} \mathrm{R}$ antagonist) and the lowest dose studied stimulated lung growth through ERK1/2 and JNK pathway. Moreover, selective activation of $\mathrm{AT}_{2} \mathrm{R}$ (using $\mathrm{AT}_{1} \mathrm{R}$ antagonist) and the highest dose studied inhibited lung branching by decreasing p-38 and JNK and increasing STAT3 phosphorylation.

Conclusions: AngII in lower doses acts through AT1R and stimulates lung growth, whereas AngII in higher doses acts through AT2R and inhibits lung branching. These results suggest AngII as a new regulator of lung morphogenesis and propose that an airwayvasculature interaction can regulate fetal lung branching. Furthermore, these actions of AngII explain why ACE-I and ARA can induce fetal lung hypoplasia after maternal administration during pregnancy. 


\section{Normocapnic hypoxia and resuscitation with $21 \% \mathrm{O}_{2}$} in newborn piglets: An experimental model

\section{F. Aroni ${ }^{\mathrm{a}}$, N. Iacovidou ${ }^{\mathrm{a}}$, T. Xanthos ${ }^{\mathrm{a}}$, A. Alexaki ${ }^{\mathrm{a}}, \mathrm{M}$. Varsami $^{\mathrm{a}}$, I. Argyri ${ }^{\mathrm{a}}$, P. Lelovas ${ }^{\mathrm{a}}$ and A. Papalois ${ }^{\mathrm{b}}$ \\ ${ }^{\mathrm{a}}$ University of Athens, Medical School, Athens, Greece \\ ${ }^{\mathrm{b}}$ ELPEN Pharmaceutical Company, Athens, Greece}

Aim: To describe a swine experimental model of normocapnic hypoxia and resuscitation.

Materials and methods: Male newborn piglets (24 days, $2.5 \pm 1.2 \mathrm{~kg}$ ) were premedicated, anaesthetized and instrumented (right carotid artery/right internal jugular vein) to continuously monitor systolic, diastolic arterial blood pressure and central venous pressure. After stabilisation, normocapnic hypoxia $\left(6-7 \% \mathrm{FiO}_{2}\right)$ was induced, followed by resuscitation with $21 \% \mathrm{FiO} 2$ when bradycardia occurred $(<60 \mathrm{bpm})$. Piglets were reoxygenated until the heart rate reached $90 \%$ of the baseline value. Arterial blood samples for biochemistry and acid-base balance were taken at stabilisation, hypoxaemia and reoxygenation.

Results: Two animals suffered asystole during hypoxia, and one was successfully resuscitated. The time needed for piglets to become bradycardic was $21.1 \pm$ 13.1min. Stabilization after reoxygenation required $30.4 \pm 22.4 \mathrm{~min}$. The variables are shown in the following table.

\begin{tabular}{|c|c|c|c|}
\hline & Baseline(B) & Hypoxaemia(H) & Reoxygenation $(\mathrm{R})$ \\
\hline $\mathrm{HR}(\mathrm{bpm})$ & $141.5 \pm 23.2$ & $50.5 \pm 10.3$ & $135.6 \pm 12.4$ \\
\hline $\begin{array}{l}\text { Mean Arterial } \\
\text { Pressure }(\mathrm{mmHg})\end{array}$ & $60.8 \pm 13.6$ & $29 \pm 9.1$ & $66.8 \pm 16.5$ \\
\hline $\mathrm{CVP}(\mathrm{mmHg})$ & $6.6 \pm 2.3$ & $9.7 \pm 3.1$ & $6.2 \pm 1.2$ \\
\hline $\mathrm{PO}_{2}$ & $102.3 \pm 18.6$ & $41.4 \pm 7.3$ & $92.5 \pm 13.0$ \\
\hline
\end{tabular}

All variables were statistically different $(B)$ vs $(H) ;(H)$ vs(R), while (B) vs(R) were NS.

Conclusion: Haemodynamic response of normocapnic hypoxic, resuscitated piglets fluctuated rendering the breed a suitable research model of asphyxia.

\section{Surfactant without mechanical ventilation - What factors are influencing the reponse to treat- ment?}

P. Jung, G. Stichtenoth, C. Härtel, W. Göpel and E. Herting

Department of Pediatrics, University of Lübeck, Germany
Introduction: Surfactant application via gastric tube in spontaneously breathing premature infants under $\mathrm{nC}$ PAP is increasingly used in Germany. Factors predicting treatment failure of this method have not been investigated so far.

Methods: We retrospectively analysed the records of 57 infants born 2004 to 2007 that were treated with surfactant in our unit by the gastric tube method. Nonresponse was defined as need for subsequent mechanical ventilation (MV).

Results: 13/57 (23\%) infants needed subsequent intubation and MV opposed to 44 responders that could be maintained on nCPAP alone following surfactant instillation under spontaneous ventilation. No differences were observed in gestational age and weight between the groups. Non-responders received less antenatal steroids and more surfactant; showed increased oxygen demand and had more complications (BPD, NEC, PDA).

Conclusion: Gestational age and weight did not predict treatment response in our study. Severe RDS and lack of antenatal steroid treatment could be factors that favour the use of conventional surfactant treatment with subsequent MV. Larger studies are needed to develop good predictive models in order to avoid the stress or repeated laryngoscopy associated with treatment failure.

\section{Routine painful procedures and oxidative stress in the newborn}

C.V. Bellieni ${ }^{\mathrm{a}}$, L. Iantorno ${ }^{\mathrm{a}}$, S. Perrone ${ }^{\mathrm{a}}$, F. Proietti ${ }^{\mathrm{a}}{ }$ A. Rodriguez ${ }^{\mathrm{a}}$, M. Longini ${ }^{\mathrm{a}}$, S. Capitani ${ }^{\mathrm{b}}$ and $\mathrm{G}$. Buonocore $^{\mathrm{a}}$

${ }^{a}$ Department of Pediatrics, Obstetrics and Reproductive Medicine, University of Siena, Siena, Italy ${ }^{b}$ Department of Pediatrics and Clinical Medicine Section of Neonatal Intensive Care Unit, University of Cagliari, Cagliari, Italy

Stressful events can damage neonatal brain through a complexity of events including free radical (FR) generation. We examined whether pain provoked by a routine heel prick (RHP) can generate an increase in potentially harmful FR in neonatal blood.

Advanced oxidative protein products (AOPP) and total hydroperoxyde $\mathrm{(TH})$ concentrations were measured at the beginning and at the end of RHP in 64 babies (corrected age: $37.3 \pm 2.5$ weeks). We scored pain of every procedure in all newborns. No increase was 
detected between AOPP and TH blood concentrations considering the whole cohort of babies. Conversely, a significant increase was observed between AOPP and TH blood concentrations considering only those babies who showed the highest grade of pain. When babies' pain was high (ABC score $\geqslant 4$ ), mean AOPP and TH blood levels increased significantly (mean AOPP values from $53.5 \mu \mathrm{m} / \mathrm{l}(\mathrm{SD}=41.6)$ to $63.2 \mu \mathrm{m} / \mathrm{l}(\mathrm{SD}$ $=44.3)$ and $\mathrm{TH}$ values from 218.3 $\mathrm{U}$ Carr $(\mathrm{SD}=89.2)$ to 228.7 U Carr (SD = 93.3); p: 0.02 and 0.036 respectively. A significant correlation was also found between AOPP blood levels ratio (sample B/sample A) in each baby, and the correspondent level of pain.

Even common routine procedures can be potentially harmful for the newborn if they provoke a high level of pain. This study adds evidence to support the use of effective analgesic procedures for every type of potentially painful event, in order to avoid the dangerous effects of FR generation.

\section{Low plasma prostanoids in extremely preterm in- fants with patent ductus arteriosus predict non- responsiveness to ibuprofen}

\author{
J. Aranda ${ }^{\mathrm{a}}$, G. Natarajan ${ }^{\mathrm{a}}$, K. Beharry ${ }^{\mathrm{b}}$, E. Capparelli ${ }^{\mathrm{c}}$, \\ R. Clyman ${ }^{\mathrm{d}}$ and R. Holt ${ }^{\mathrm{e}}$ \\ aPPRU, Children's Hospital, Detroit, MI and State \\ University of New York Downstate Medical Center, \\ Brooklyn, NY, USA \\ ${ }^{\mathrm{b}}$ University of California, Irvine, CA, USA \\ ${ }^{c}$ PPRU, University of California, San Diego, CA, USA \\ ${ }^{\mathrm{d}}$ University of California, San Francisco, CA, USA \\ ${ }^{\mathrm{e}}$ University of Illinois and Ovation Pharma Chicago, \\ IL, USA
}

Background: Prostanoids (PDs) exert contractile or relaxant actions on the ductus arteriosus.

Objectives: To determine plasma prostanoid profiles in the first days of life and changes in response to ibuprofen and to compare these among infants who responded to ibuprofen and those who needed rescue treatment.

Methods: A subset of 74 preterm neonates (38 placebo and 36 ibuprofen-treated) of the multicenter randomized double-blind placebo controlled ibuprofen trial underwent blood sampling before, 1 hour after the first dose, and 1 hour after the third dose (73 hours) of ibuprofen or placebo. Plasma prostanoids (PG) $\mathrm{E}_{2}$, $\mathrm{PGF}_{2 \alpha}, 6$-ketoPGF $1 \alpha$ and thromboxane (Tx) $\mathrm{B}_{2}$ were assayed by ELISA.
Results: Baseline plasma prostanoids were higher than reported for adults and children and remained elevated albeit with considerable variations through the study period. $\mathrm{TxB}_{2}$ levels were significantly elevated in the placebo group at all time points compared to ibuprofen-treated infants. Ibuprofen had no significant effect on prostanoid concentrations, nor did plasma ibuprofen levels correlate with plasma prostanoid levels. Infants who required rescue treatment had significantly lower $\mathrm{PGE}_{2}, 6-\mathrm{ketoPGF}_{1 \alpha}$ and $\mathrm{TxB}_{2}$ at baseline, as well as lower $\mathrm{PGE}_{2}$ and 6-ketoPGF $1 \alpha 1$ hour after the first dose, compared to infants who responded to ibuprofen.

Conclusions: Extremely preterm infants who do not respond to ibuprofen have lower circulating prostanoid levels than responders. Low prostanoid levels may result in immature ductal remodeling and inability to contract even with prostanoid inhibitors.

\section{Diagnosis and cause of death in a NICU - How im- portant is autopsy?}

S. Costa ${ }^{\mathrm{a}}$, M. Rodrigues ${ }^{\mathrm{a}}$, O. Brandão ${ }^{\mathrm{b}}$, A. Martins ${ }^{\mathrm{a}}$, A. Vilan ${ }^{\mathrm{a}, \mathrm{c}}$ and H. Guimarães ${ }^{\mathrm{a}, \mathrm{c}}$

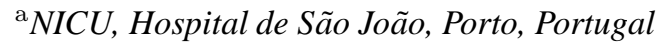

${ }^{\mathrm{b}}$ Pathology Department, Hospital de São João, Porto, Portugal

${ }^{\mathrm{c}}$ Faculty of Medicine, Porto, Portugal

Introduction: Autopsy has been important in medicine, not only in determining cause of death, but also as a tool in clinical education and quality monitoring in patient care. However, general autopsy rates have declined in recent years.

Objectives: Characterize mortality in a tertiary referral Neonatal Intensive Care Unit (NICU) in Portugal, measure the neonatal autopsy rate and determine the concordance between ante-mortem and post-mortem diagnosis.

Material and Methods: Retrospective review of the clinical and pathology records of infants who died in five consecutive years (2004 to 2008). For those with autopsies, pathology and clinical diagnosis were compared and classified according to general concordance and to the modified Goldman classification.

Results: During the referred period, 1938 patients were admitted to the NICU. The mortality rate was $5.7 \%$ (110 patients). The age at death varied from 1 to 317 days (mean 24.3). 
53 patients were submitted to autopsy, performing a $48.2 \%$ global autopsy rate (minimum $34.8 \%$ in 2005 and maximum $60 \%$ in 2008).

The most frequent causes of death were congenital malformations and prematurity with its complications,.

Pathology agreed with the clinical diagnosis in 18 cases $(34 \%)$ and additional findings were discovered in 22 newborns (42\%); in 13 cases $(24.5 \%)$ the clinical diagnosis was revised or the diagnosis was established by pathology. Five autopsies revealed information relevant for genetic counselling.

The most frequently omitted diagnosis was pneumonia; sepsis was the condition most frequently overdiagnosed.

Conclusion: Despite the high agreement rate between clinical and pathology diagnosis, autopsy frequently added important data, including several cases in which it established the diagnosis or provided relevant information for parental counselling regarding future pregnancies.

\section{Neutropenia, systemic infection and mortality in preterm, small for gestational age neonates}

\author{
R. Carr ${ }^{\mathrm{a}}$, C. Doré ${ }^{\mathrm{b}}$, P. Brocklehurst ${ }^{\mathrm{c}}$ and N. Modi ${ }^{\mathrm{d}}$ \\ ${ }^{\mathrm{a}}$ Guy's Hospital, King's College London, UK \\ ${ }^{\mathrm{b}}$ MRC Clinical Trials Unit, London, UK \\ ${ }^{\mathrm{c}}$ National Perinatal Epidemiology Unit, University of \\ Oxford, UK \\ ${ }^{\mathrm{d}}$ Imperial College, Chelsea \& Westminster campus, \\ London, UK
}

Background: Systemic infection (SI) is frequent in preterm babies. Neutropenia is believed to increase the risk. The PROGRAMS trial ${ }^{1}$ assessed whether prophylactic GM-CSF would reduce sepsis and mortality. Trial outcome showed that GM-CSF corrected neutropenia, but sepsis and mortality were equal in treated and control subjects.

This analysis utilises the haematological, clinical and microbiological data collected over 28 days from recruitment to examine the impact of neutropenia and SI on pre-discharge mortality.

Methods: SI diagnosis was based on microbiological cultures, platelet count, CRP, and occurrence of 12 clinical signs associated with acute-onset SI. Neutropenia defined as ANC $\leqslant 1.0 \times 10^{9} / 1$.

Results: 280 infants $<32$ weeks gestation and birthweight $<10$ th centile were recruited from $26 \mathrm{UK}$ neonatal units. Infants experiencing neutropenia had an increased incidence of SI 63/127(50\%) v no neutropenia 51/153(33\%) [ $p=0.006]$. Neutropenic infants had increased mortality: neutropenia 40/127(32\%) v no neutropenia $22 / 153(14 \%)[p=0.001]$. Infants who experienced one or more episodes of SI had increased mortality: SI 46/114(40\%) v no SI 16/166(10\%) [ $p<$ 0.001]. Mortality in the 102 infants who had neither neutropenia or sepsis during the study period was only $6 \%$.

Conclusions: In these high risk infants the occurrence of both neutropenia and systemic infection independently increased the risk of all cause mortality prior to discharge.

\section{Grateful for life: Your son's and your own}

L. Cataldi ${ }^{\mathrm{a}}$ and M.G. Gregorio ${ }^{\mathrm{b}}$

${ }^{a}$ Department of Pediatrics, Division of Neonatology, Catholic University School of Sacred Heart, Rome, Italy

${ }^{\mathrm{b}}$ Pediatric Heath Service, ASL 8, U.O.C.C., Cagliari, Italy

"Donaria" is the name by which the ancients designated presents made to the gods, either by individuals or communities. Sometimes they are also called dona or $\delta \tilde{\omega} \rho \alpha$. Presents were mostly given as gratitude's token for some favour which a god had bestowed on man. The belief that the gods were pleased with costly presents was as natural to the ancients as the belief that they could be influenced in their conduct towards men by the offering of sacrifices; and, indeed, both sprang from the same feeling. the "donaria" usually represented the full body or the part of them (head, arm, leg, feet) or different organs (breast, uterus, gut, genital organs, eyes, ears, etc.).

The custom of making donations to the gods is found among the ancients from the earliest times of which we have any record; even after the introduction of Christianity it was, with some modifications, observed during the middle ages.

In more recent centuries, starting in Italy, in 15 th century, ex-votos, a devotional painting giving thanks to a saint or deity for a miraculous healing or a blessing, continued to be a popular expression of faith and healing into the late 18 th and early 19 th centuries. The faithful have always used prayer to invoke the aid of saints as a means to heal the sick and end one's suffering. have played an important role in the daily lives.

Ex-votos continue to reflect a tradition where faith and healing play a vital role in daily life. 
Authors present and comment some ex-voto pictures collected around.

\section{Effect of circulating angiotensin converting enzyme (ACE) on diaphragmatic strength in infants}

G. Dimitriou ${ }^{\mathrm{a}}$, D. Papakonstantinou ${ }^{\mathrm{a}}$, E. Stavrou ${ }^{\mathrm{b}}$, S. Tzifas ${ }^{\mathrm{a}}$, A. Onufriou ${ }^{\mathrm{c}}$, A.Athanassiadou ${ }^{\mathrm{b}}$ and S. Mantagos ${ }^{\mathrm{a}}$

${ }^{a}$ Neonatal Intensive Care Unit, Department of Paediatrics, University Hospital of Patras, Patras, Greece

${ }^{\mathrm{b}}$ Department of General Biology, School of Medicine, University of Patras, Patras, Greece

${ }^{\mathrm{c}}$ Department of Biochemistry, University Hospital of Patras, Patras, Greece

Background: The human ACE gene contains a polymorphism consisting of either the presence (I) or absence (D) of a 287-base fragment and partially determines circulating ACE (cACE) levels. Adult studies have suggested an association of the cACE in the regulation of skeletal muscle strength. Diaphragmatic strength in infants can be assessed by measuring transdiaphragmatic pressure during crying $\left(\mathrm{Pdi}_{\max }\right)$.

Aim: To examine the relationship between cACE activity and diaphragmatic strength in infants. Patients: One hundred infants, median gestational age 36 (2840) weeks, were studied.

Methods: ACE genotyping was performed by PCR amplification. ACE activity was assayed using a UVkinetic method. Pdi $\mathrm{Pax}_{\max }$ was measured using a pressure catheter during airway occlusions performed at the end of a crying effort.

Results: Pdi $\mathrm{max}_{\max }$ indexed to body weight was positively related to cACE levels $(p=0.025)$ independent of postmenstrual age and gender, but not to ACE gene polymorphism.

Conclusion: These results suggest that the cACE levels may affect diaphragmatic strength in infants.

\section{Respiratory support strategies for the preterm new- born - National survey (2008)}

G. Rocha ${ }^{\mathrm{a}}$, J. Saldanha ${ }^{\mathrm{b}}$, I. Macedo ${ }^{\mathrm{c}}$, A. Areias ${ }^{\mathrm{d}}$ and T. Toméc

a Serviço de Neonatologia, São João Hospital, Porto, Portugal

${ }^{\mathrm{b}}$ Unidade de Neonatologia, Hospital de Santa Maria, Lisbon, Portugal
${ }^{\mathrm{c}}$ Unidade de Neonatologia, Maternidade Dr. Alfredo da Costa, Lisbon, Portugal

dUnidade de Neonatologia, Maternidade Júlio Dinis, Centro Hospitalar do Porto, Porto, Portugal

Background: Respiratory support strategies for the preterm newborn have been a subject of intense research.

Aim: To survey current practice in Portugal regarding neonatal respiratory support.

Methods: An electronic questionnaire survey of 31 Portuguese neonatal units was undertaken to assess the respiratory support practices used in the preterm newborn.

Results: There was a 94\% response rate. Draeger babylog is the most frequently used ventilator. Twelve $(41 \%)$ units prefer the use of early NCPAP whenever possible. When invasive ventilation is needed, triggered ventilation is the choice of $24(83 \%)$ units (SIMV, SIPPV, PSV); four (14\%) units have the possibility of HFOV. SIMV is the most frequent ( $n=13.45 \%$ ) weaning mode. Exogenous surfactant is used as prophylactic in $12(41 \%)$. All units use lung function measurements to aid choice of ventilator settings. Seventeen (59\%) units allow oxygen saturation levels from $90 \%-$ $95 \%$ for infants with acute respiratory disease and 15 (52\%) levels from $85 \%-90 \%$ in infants with chronic lung disease.

Conclusions: Many of the respiratory strategies for the ELBW infant reflect the result of large randomised trials. More effective methods may be required to encourage the use of early NCPAP, the use of SIPPV as the main weaning mode, the use of volume targeted ventilation and a more judicious use of oxygen and exogenous surfactant in order to ensure evidence - based practice.

Perinatology and prematurity in developing countries

\section{J. Haddad}

Neonatology, St George University Hospital Beirut, Lebanon

Despite several conventions on child's rights, neonatal mortality and morbidity are still a major problem in developing countries particularly for prematures. To assess the problematic of prematurity in Lebanon, we have underwent in collaboration with UNICEF and the Ministry of Health a national study, all over the coun- 
try during 2006 and 2007 on perinatal mortality and morbidity.

Results:

- incidence of prematurity $9 \%$ compared to France $7 \%$

- incidence of IUGR 7\% as in France

- incidence of Stillbirth $1.9 \%$ compared to $0.5 \%$ in France

- for prematures $<28$ WGA rates of stillbirth, neonatal mortality $<7$ days and perinatal mortality are respectively $66 \%, 66 \%$ and $88 \%$

- regarding prematures between 28 and 32 WGA the rates are respectively $32 \%, 17 \%$ and $44 \%$

- concerning neonates of 33 to 36 WGA the rates decrease to $9.6 \%, 8.7 \%$ and $17.8 \%$

- neonatal mortality is estimated at $27 \%$

- Child mortality is around 28\%, $70 \%$ occurring in the neonatal period.

We divided the problematic of prematurity into three components:

Limits of viability: We were faced to the crucial question of resuscitating aggressively prematures $<28$ WGA. Furthermore reports from the literature concerning this population is not enthusiastic, sequels being a major handicap and follow up a big gap.

Modern techniques, morbidity and cost: in Developing countries the introduction of such technics is not denied of side effects (surfactant, HFO, ECMO, Antibiotics, Oxygen and steroids). An appropriate education, a quality assurance evaluation and transparency must accompany initiation of modern techniques.

The organization of care: in Lebanon more than 170 establishments are authorized to undergo deliveries for a population of 3 million inhabitants. There is no level nor regionalization of care. Thus, the absence of such a strategy seems to be significantly related to mortality and morbidity.

Difficult airway - How the problem is recognised in Polish neonatal units and paediatric intensive care units

T. Szreter ${ }^{\mathrm{a}}$, M. Migdal ${ }^{\mathrm{a}}$, J. Zejda ${ }^{\mathrm{b}}$, G. Brozek ${ }^{\mathrm{b}}$, E. Musialik-Swietlinska $^{c}$ and J. Swietlinski ${ }^{\mathrm{a}}$

${ }^{a}$ Department of Anaesthesiology and Intensive Care, Children's Memorial Health Institute, Warsaw, Poland ${ }^{\mathrm{b}}$ Department of Epidemiology, Silesian University of Medicine, Katowice, Poland

${ }^{\mathrm{c}}$ Department of Neonatal Intensive Care, Silesian University of Medicine, Katowice, Poland
Aim: To assess existing currently in Poland knowledge on difficult airway problem.

Methodology: The study was performed as a part of "The National Survey on Practices in Paediatric and Neonatal Intensive Care" in 2008. The questionnaire referred to difficult airway was sent to all Polish neonatal units (416 centers) and pediatric intensive care units (53 centers). Data collection and statistical analysis was performed by the independent centre.

Results: The overall response rate was $65 \%$. Recognition of difficult airway problem was reported by $93 \%$ PICUs and $32 \%$ neonatal units $(p=0.0003)$. Assessment of degree of airway obstruction and functional impairment in children at risk was declared by $79 \%$ PICUs and $40 \%$ neonatal units $(p=0.001)$.

Conclusions: Results of the survey confirmed need for better education on difficult airway problem especially among medical staff of neonatal units in Poland.

\section{Auditory ERPS at preschool age are different after extremely preterm birth and correlated to cognitive performance}

V. Fellman ${ }^{\mathrm{a}}$, H. Hövel ${ }^{\mathrm{a}}$, I. Rosén ${ }^{\mathrm{b}}$, L. HellströmWestas $^{\mathrm{a}}$, K. Stjernqvist ${ }^{\mathrm{c}}$ and M. Huotilainen ${ }^{\mathrm{d}}$

${ }^{a}$ Department of Pediatrics, Lund University, Lund, Sweden

${ }^{\mathrm{b}}$ Department of Neurophysiology, Lund University, Sweden

${ }^{\mathrm{c}}$ Department of Psychology, Lund University, Lund, Sweden

${ }^{\mathrm{d}}$ Cognitive Brain Research Unit, Department of Psychology, University of Helsinki, Finland

In children, pathological auditory event-related potentials (aERP are associated with cognitive dysfunction. We studied whether aERP (particularly Mismatch negativity, MMN at 200-350 ms after stimuli) in extremely preterm infants (EP, $n=71,27.5 \pm 1.9 \mathrm{gw}$, at 5 years or age are different from preterm $(n=12$, $33.7 \pm 1.0 \mathrm{gw})$ and term-born controls $(n=15)$, and related to cognition.

A 75-ms harmonic standard stimulus was presented with three deviants pseudo-randomly at $10 \%$ probability each, different in frequency (10\% higher), perceived sound-source location (PSSL), or sound duration (25 ms). Intelligence quotient (IQ) was tested with WPPSI-R.

For standard and all deviant ERPs, EP had lower amplitudes and shorter latencies both for P120 and the 
following N250. A MMN for the PSSL deviant occurred only in $34 \%$ of EP and $37 \%$ of controls. EP had lower WPPSI-R full-scale and subscale IQ than the other groups.

Processing speed IQ correlated with the latency of the Standard P120 peak $(\mathrm{R}=0.359 / 0.393$ and $p=$ $0.001 /<0.001$ for frontal/central electrodes, respectively).

ERPs showed consistent group differences that may imply a higher sound sensitivity of EP which may contribute to their cognitive problems.

Ontogeny of drug glucuronidation in neonates: Extensive interindividual variability despite their young age

K. Allegaert ${ }^{\mathrm{a}}$, J. de Hoon $^{\mathrm{b}}$ and G. Naulaers ${ }^{\mathrm{a}}$

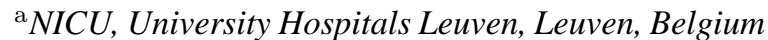

${ }^{\mathrm{b}}$ Center for Clinical Pharmacology, University Hospitals Leuven, Leuven, Belgium

Background and aims: Data on iso-enzyme specific phenotypic activity of uridine diphosphate glucuronosyltransferase (UGT) and its covariates are limited, but of clinical relevance when pharmacokinetics, pharmacodynamics and toxicology in neonates are considered. We therefore wanted to described the UGT ontogenic pattern based on paracetamol, tramadol and propofol metabolism in neonates.

Methods: The contribution of glucuronidation to overall renal drug elimination after tramadol, paracetamol or propofol administration was quantified based on metabolic ratio's in $24 \mathrm{~h}$ urine collections in neonates.

Results: For all three probe drugs evaluated, extensive interindividual variability was observed. Besides postmenstrual age, there was a significant contribution of postnatal age on the phenotypic activity observed. Finally, during repeated administration, there was a further increase in the phenotypic activity observed. However, based on the covariates mentioned, only about $60-65 \%$ of the interindividual variability observed can be explained.

Conclusions: Postmenstrual and postnatal age only in part explain the interindividual variability observed. A focused approach to unveil additional covariates of the interindividual range (e.g. disease state characteristics, genetic polymorphisms) is needed to further improve our knowledge on the covariates of UGT ontogeny and drug disposition in early life.
Neurodevelopmental outcome in the third year of life after intrauterine laser coagulation for severe twin-twin-transfusion syndrome (TTTS)

P. Bartmann ${ }^{\mathrm{a}}$, K. Hecher ${ }^{\mathrm{b}}$, S. Bektas ${ }^{\mathrm{a}}$, C. Banek ${ }^{\mathrm{a}}$, C. Maschke $^{\mathrm{a}}$, B. Ellenrieder ${ }^{\mathrm{a}}$ and A. Diemert ${ }^{\mathrm{b}}$

${ }^{a}$ University of Bonn, Department of Neonatology, Bonn, Germany

${ }^{\mathrm{b}}$ University Medical Center Hamburg-Eppendorf, Department of Obstetrics and Fetal Medicine, Hamburg, Germany

Objective: To evaluate the neurodevelopmental outcome in three consecutive groups of patients after intrauterine laser coagulation for severe TTTS.

Patients and Methods: All patients received prenatal treatment in Hamburg and follow-up examinations in Bonn. 89/89 survivors of the treatment period Jan 95May 97, 167/172 from June 97-Sept 99 and 128/179 from Jan 04-Sept 06 were evaluated in their third year of life by standardized neurological examinations and neurodevelopmental tests.

Results: Survival after laser coagulation as well as developmental outcome improved significantly from the first to the latest time period.

\begin{tabular}{ccccc}
\hline $\begin{array}{c}\text { Treatment } \\
\text { period } \\
\text { (year) }\end{array}$ & $\begin{array}{c}\text { Overall } \\
\text { survival } \\
(\%)\end{array}$ & $\begin{array}{c}\text { Normal } \\
\text { development } \\
(\%)\end{array}$ & $\begin{array}{c}\text { Low- } \\
\text { moderate } \\
\text { handicap }(\%)\end{array}$ & $\begin{array}{c}\text { Severe } \\
\text { handicap } \\
(\%)\end{array}$ \\
\hline $1 / 95-5 / 97$ & 61.0 & 77.5 & 11.2 & 11.2 \\
$6 / 97-9 / 99$ & 67.7 & 86.8 & 7.2 & 6.0 \\
$1 / 04-9 / 06$ & 81.5 & 89.8 & 7.8 & 2.3 \\
\hline
\end{tabular}

Conclusion: There is evidence from these data that improvement in survival after intrauterine laser coagulation for severe TTTS is accompanied by improvement in neurodevelopmental outcome in the third year of life.

Isoprostanes levels in urine of preterm newborns treated with ibuprofen for PDA closure

S. Perrone ${ }^{\mathrm{a}}$, M. Longini ${ }^{\mathrm{a}}$, R. Antonucci ${ }^{\mathrm{b}}$, V. Iantorno, F. Proietti ${ }^{\mathrm{a}}$, A. Rodriguez ${ }^{\mathrm{a}}$, B. Marzocchi ${ }^{\mathrm{a}}$, V. Fanos ${ }^{\mathrm{b}}$ and G. Buonocore ${ }^{\mathrm{a}}$.

${ }^{a}$ Department of Pediatrics, Obstetrics and Reproductive Medicine, University of Siena, Siena, Italy

${ }^{\mathrm{b}}$ Department of Pediatrics and Clinical Medicine Section of Neonatal Intensive Care Unit, University of Cagliari, Cagliari, Italy 
Ibuprofen (IBU), an alternative non steroid antinflammatory drug (NSAID), has been proposed for the treatment of PDA in the newborn due to the lower reduction in cerebral, renal and mesenteric blood flow when compared with indomethacin.

The present study tests the hypothesis that an OS may occur in neonate with PDA. Secondary we verified whether IBU for PDA therapy induces changes in urinary isoprostane (iPs) levels.

Thirty five preterm babies $<33$ weeks were studied prospectively. Three urine samples were collected: before and after IBU treatment and 7 days after the end of treatment

No differences were found between urinary levels of iPs at time 0 . A statistically significant difference was found in iPs time behaviour between newborns treated with IBU and control ones (Kruskal-Wallis: $p=0.04$ versus $p=\mathrm{NS}$ ).

Time trend showed a significant decrease in iPs from time 0 to time 1 after IBU therapy ( $p=0.03$ ) followed by an increase in iPs levels 7 days after the treatment $(p=0.04)$. No difference existed in control group. IBU therapy for PDA closure showed to reduce the risk of OS related to free radical generation. This antioxidant effect of IBU may be beneficial in pre term babies with PDA who are at high risk for OS. The results of the present study pave the way for more larger randomized clinical trials to confirm the antioxidant effect of IBU therapy.

Spontaneous respiratory rate response to expiratory tidal volume setting in an experimental model with normal lung and after bronchoalveolar lavage (BAL)

M. Sanchez Luna ${ }^{\mathrm{a}}$, F. J. Tendillo ${ }^{\mathrm{b}}$ and M. Santos ${ }^{\mathrm{b}}$

${ }^{a}$ Neonatal Division, Hospital Universitario MaternoInfantil Gregorio Marañón, Complutense University, Madrid, Spain

${ }^{\mathrm{b}}$ Department of Experimental Surgery, University Hospital Puerta de Hierro Majadahonda, Madrid, Spain

Background: The spontaneous breathing rate (SRR) of a patient in response to a preset $\mathrm{V}_{T}$ during volume guarantee ventilation (VG) combined with flow cycled ventilation, (PSV) could represent an adequate $\mathrm{V}_{T}$ setting, and probably correlates with a normal $\mathrm{PaCO}_{2}$ range.

Objective: To investigate the effect of different $\mathrm{V}_{T}$ settings on spontaneous respiratory rate (SRR), respiratory rate/tidal volume ratio (RVR) and lung mechanics.
Design/Methods: Six newborn piglets were ventilated with a Dräger BB8000+ ventilator in PSV with spontaneous breathing and VG initially set at $4 \mathrm{ml} / \mathrm{kg}$ of $\mathrm{V}_{T}$ and increased in $10 \mathrm{~min}$ intervals in $1 \mathrm{ml} / \mathrm{kg}$ until a $V_{T}$ of $10 \mathrm{ml} / \mathrm{kg}$. with normal lungs and after artificial RDS induced with a BAL. Respiratory mechanics, heart rate and blood pressure were continuously recorded and arterial blood gas measured after each $10 \mathrm{~min}$ intervals.

Results: SRR, RVR and $\mathrm{PaCO}_{2}$ decreased after increase in $\mathrm{V}_{T}$, in normal lung $(p<0.05)$. In the RDS lung, SRR, RVR and $\mathrm{PaCO}_{2}$ increased by decreasing $\mathrm{V}_{T}$ settings $(p<0.05)$. Minute volume inversely correlated with SRR $(p<0.05)$.

Conclusions: In this animal model, during PSV with VG ventilation in normal lungs and after BAL, changes in $\mathrm{V}_{T}$ induced inverse changes in the SRR. The SRR and RVR value could be an important guide to set expiratory $\mathrm{V}_{T}$ during VG with PSV.

\section{The profile of early pathology in late preterm infants} (LPI)

E. Kostopoulou, L. Kovacs and A. Papagerorgiou Department of Neonatology, Jewish General Hospital, McGill University, Montreal, Canada

Background: Infants born at late preterm gestational ages account for approximately $70 \%$ of all preterm births and are at risk for early and long-term morbidity.

Objective: To assess the incidence of pathology and the need and type of early medical interventions among LPI (34 0/7 to 36 6/7 weeks).

Methods: We analysed the electronic data of all LPI born in our tertiary care perinatal center who needed admission to the NICU during the period of 2003-2007 (5 years). Infants with anomalies incompatible with life were excluded from the analysis.

Results: 585 LPI born during the 5 year period, $310(52.9 \%)$ males and $275(47.1 \%)$ females, required admission. Table 1 indicates the most significant reason for admission.

Conclusions: Our data confirms the fact that late preterm deliveries carry a significant risk for neonatal morbidity. These newborns require careful evaluation soon after birth and quite frequent admissions to the NICU for specialized care. 


\begin{tabular}{lllcc}
\hline & 34 weeks & 35 weeks & 36 weeks & Total \\
\hline NICU admissions & $\mathrm{N}=304$ & $\mathrm{~N}=173$ & $\mathrm{~N}=108$ & $\mathrm{~N}=585$ \\
& $(51.9 \%)$ & $(29.6 \%)$ & $(18.5 \%)$ & $(100 \%)$ \\
Need for $\mathrm{O}_{2}$ & $68(22.4)$ & $44(25.3)$ & $29(26.8)$ & $141(24.1)$ \\
RDS & $34(11.2)$ & $17(9.8)$ & $11(10.2)$ & $62(10.6)$ \\
CPAP & $38(12.4)$ & $23(13.2)$ & $15(13.8)$ & $76(13.0)$ \\
Mechanical & $21(6.9)$ & $14(8.0)$ & $8(7.4)$ & $43(7.3)$ \\
ventilation & & & & \\
Surfactant & $10(3.3)$ & $8(4.6)$ & $5(4.6)$ & $23(3.9)$ \\
TTN & $36(11.8)$ & $21(12.1)$ & $12(11.1)$ & $69(11.8)$ \\
\hline
\end{tabular}

${ }^{1}$ H NMR-based metabolomic analysis of urine from term and preterm neonates

R. Antonucci ${ }^{\mathrm{a}}$, L. Barberini ${ }^{\mathrm{b}}$, R. Defraia ${ }^{\mathrm{a}}$, R. Agostiniani $^{\mathrm{c}}$, E. Locci ${ }^{\mathrm{d}}$, P.Cortesi ${ }^{\mathrm{c}}$, F. Cesare Marincola ${ }^{\mathrm{d}}$, P. Scano $^{\mathrm{d}}$, A. Lai ${ }^{\mathrm{d}}$, L. Atzori ${ }^{\mathrm{e}}$ and V. Fanos ${ }^{\mathrm{a}}$

${ }^{a}$ Neonatal Intensive Care Unit, University of Cagliari, Cagliari, Italy

${ }^{\mathrm{b}}$ Department of Neurological Sciences, University of Cagliari, Cagliari, Italy

${ }^{\mathrm{c}}$ Pediatric Division, Pescia Hospital, Cagliari, Italy

${ }^{\mathrm{d}}$ Department of Chemical Sciences, University of Cagliari, Cagliari, Italy

${ }^{\mathrm{e}}$ Department of Toxicology, University of Cagliari, Cagliari, Italy

Background: Metabolomics is a general approach to determining a picture of the current metabolic status of an organism by measuring hundreds or thousands of metabolites simultaneously in an intact tissue or biofluid.

Aim: To analyze urinary metabolic profiles in term and preterm infants in order to identify metabolic patterns associated with different gestational ages.

Methods: Twenty-six healthy term infants (gestational age $=39.7 \pm 0.8 \mathrm{wks}$; birth weight $=3292$ $\pm 364 \mathrm{~g}$ ) and 41 preterm infants (gestational age $=$ $32.0 \pm 3.0 \mathrm{wks}$; birth weight $=1830 \pm 758 \mathrm{~g}$ ) were prospectively enrolled. For each patient, a urine sample was collected non-invasively in the first 12 hours of life. The samples were stored frozen until analysis by Proton Nuclear Magnetic Resonance $\left({ }^{1} \mathrm{H}\right.$ NMR) spectroscopy. Data from NMR spectra of urine samples were statistically analyzed by using Principal Components Analysis and Partial Least Squares-Discriminate Analysis (SIMCA software).

Results: The multivariate statistical analysis of spectral data identified different classes of neonates that were characterized by different metabolic patterns. Term infants differed significantly from preterm infants. Preterm infants of 23-32 weeks' gestation differed significantly from those of 33-36 weeks' gesta- tion. Metabolites discriminating between groups were hyppurate, tryptophane, phenylalanine, malate, tyrosine, hydroxybutyrate, N-acetyl-glutammate, proline.

Conclusions: Metabolomic analysis demonstrated different urinary metabolic profiles in neonates of different gestational ages. This technique may prove to be of use in the diagnosis of pathological conditions, in evaluating drug response, in monitoring therapy or in predicting the onset of renal disorders in later life.

\section{Severe intrauterine growth restriction (IUGR) - Growth and development evaluation}

G. Pereira, B. Sampaio, C. Sá, A. Silva, E. Abreu, A. Cadilhe, J. Matos Cruz and A. Pereira São Marcos Hospital, Braga, Portugal

Introduction: Newborns with IUGR had suffered one anoxia that disturbed their growth.Cognitive impairment, motor delay, language disabilities and behaviour problems can occur

Purpose: Growth and development evaluation of prenatal diagnosed severe IUGR.

Material and Methods: Descriptive and prospective study of severe IUGR, percentile of fetal growth $<3$ in unifetal pregnancy, diagnosed since July 1 st 2005 to June 30th 2006. Maternal risk factors, placental disorders and neonatal morbidity. Growth evaluation using the National Centre for Health and Statistics growth charts and development evaluation with the Denver II scale and Paediatric Evaluation Development Status (PEDS). Follow-up until 3rd year.

Results: 14 fetuses with severe IUGR. Maternal age $30.4 \pm 6.4$ years. Smokers $4 / 14$, arterial hypertension $4 / 14$, gestational diabetes $1 / 14$. Median diagnostic gestational age 33 weeks. 5/12 placentas showed infarcts and $2 / 12$ revealed infection. Median gestational age at birth 33.5 weeks. Symmetric IUGR 13/14. Very low weight $8 / 14$. The most common neonatal diagnosis were prematurity and jaundice. Follow-up on 13/14. Average current age $31 \pm 4.7$ months. Persistence of weight, height and head circumference $\mathrm{P}<52 / 13$ and weight $<$ P5 2/13.

Sat without support $7.8 \pm 2.6$; walk independently $14.5 \pm 4.5$, first words $13.8 \pm 4$, first simple phrases $20.6 \pm 4.6$ months. Gross motor impairment 3/13, fine motor impairment $1 / 13$ and language problems $2 / 13$. No social behavior impairment.

3/13 parents showed concerns on PEDS.

Conclusion: Currently, average age 31 months, 4/13 with persistent weight $<$ P5. Development impairment 
reported on 3 areas: language, gross and fine motor. The parents who have affected children are worried about that development delay, according PEDS.

Cranial ultra-sonography (CUS) and transfontanellar Doppler in premature neonates (24-32 weeks of gestation WG): Dynamic evolution during their neonatal hospital stay in the aquitaine cohort, 20032005

O. Brissaud ${ }^{\mathrm{a}}$, J.L. Demarquez ${ }^{\mathrm{a}}$, A. Bouvet ${ }^{\mathrm{b}}$, S. Boufkhed $^{\mathrm{b}}$, L. Joly-Pedespan ${ }^{\mathrm{a}}$ and V. Leroy ${ }^{\mathrm{b}}$

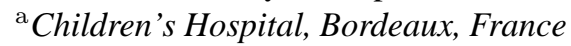

${ }^{\mathrm{b}}$ Victor Segalen Bordeaux 2 University, Bordeaux, France

Background: We investigated by CUS, the hemodynamic and morphological cerebral lesions evolution a in a cohort of premature newborn during their neonatal hospital stay.

Material and Methods: 452 newborns born under $32+6 \mathrm{WG}$ were included in this prospective cohort study. During their neonatal hospital stay, standardized CUS coupled with Doppler were made early $(<$ Day-8) and late (Month-1 - Month-2). A CUS anomaly was defined by the presence of at least one morphologic lesion (intra-ventricular haemorrhage (IVH) all stages, periventricular leukomalacia (PVL) or hyperechogenicity (flares) of the white matter at Month 1-2).

Results: The number (\%) of babies who presented respectively an IVH I, II, III and IV was: $87(19.2 \%)$, $19(4.2 \%), 10(2.2 \%)$ and $6(1.3 \%) ; 16$ neonates $(3.5 \%)$ presented PVL, 244 (54\%) persistent flares; 290 infants (64.2\%) presented at least one morphological lesion on CUS. Multivariate analysis highlights that related factors to late CUS abnormality or a death linked to neurological impairment are successively: early CUS abnormality, very high prematurity and a thrombopenia occurrence during hospitalization. In our study, Doppler velocimetry in neonates with late CUS abnormalities seems to remain stable but with no statistical difference with neonates without late CUS abnormalities.

Conclusions: Our results are in accordance with literature concerning the CUS neonates under than 33 WG' abnormalities. The Doppler velocimetry' stability in this population may reflect a risk for late CUS lesion and could be considered as a marker to decide to realise a MRI/DWI.
Birth at the borderline of viability: Guidelines from the French neonatal society

G. Moriette ${ }^{\mathrm{a}}$, S. Rameix, E. Azria, A. Fournié, P. Andrini, L. Caeymaex, C. Dageville, F. Gold, P. Kuhn, L. Storme and U. Siméoni

a Port-Royal, Paris, France

Introduction: Following birth at the margin of viability, an immediate decision has to be made between 2 options. 1) Institution of full intensive care benefits to many infants, but some infants will be severely disabled. 2) Institution of palliative care lets babies die, but some of them would have developed normally following intensive care. A working group of the French neonatal society addressed these issues.

General considerations: Birth at 24 and 25 weeks of post-menstrual age was defined as a "grey" zone of major prognosis uncertainty, in which decisions should be made in partnership with parents, based on the infant best interest. In addition to post-menstrual age, the individual decision should take into account data which modify the prognosis (birth weight, single or multiple pregnancy, sex, antenatal corticosteroids, birth in a tertiary centre...).

Guidelines:

- Any decision to withhold intensive care at birth should be taken in agreement with the 2005 law.

- At 26 or 27 weeks full intensive care should usually be instituted.

- At 25 weeks, full intensive care should be instituted, unless unfavourable factors are associated. Decision at birth should be made by a senior neonatologist, based on both the baby's condition and the parent wishes.

- At 24 weeks, the parent wishes should be followed for deciding between the institution of full intensive care and that of palliative care.

- Below 24 weeks, palliative care is the only option to be offered at the present time.

Newborns of medical assisted reproduction: Are they different?

G. Vasconcellos ${ }^{\mathrm{a}}, \mathrm{S}$. Correia ${ }^{\mathrm{b}}$, L. Calejo ${ }^{\mathrm{c}}$,

M. Rodrigues ${ }^{\mathrm{a}}$ and H. Guimarães ${ }^{\mathrm{a}}$

${ }^{a}$ Neonatology Service, Hospital S. João, University of Porto Medical School, Porto, Portugal

${ }^{\mathrm{b}}$ Department of Hygiene and Epidemiology, Institute of Public Health (ISPUP), Porto, Portugal 
${ }^{\mathrm{c}}$ Obstetric Service, Hospital S. João, University of Porto Medical School, Porto, Portugal

Introduction: The use of assisted reproductive techniques (ART) to treat infertility is increasing rapidly. Research on short and long term health effects should be conducted in specific settings.

Objective: To compare neonatal data from spontaneous and ART pregnancies stratified by single and multiple births.

Material and methods: We used data from 7780 newborns of a birth cohort (Generation XXI), assembled in 2005-2006 in 5 level III maternities. We compared perinatal outcomes between spontaneous and ART pregnancies, stratified by the number of fetus, using the qui-square test.

Results: The proportion of children born by ART was $1.4 \%$. Multiple birth were more frequent among ART $(17.1 \%$ vs. $1.32 \%, p<0.001)$.

In single ART births, the proportion of mothers over 34 years was higher $(27.8 \%$ vs. $15.7 \%, p<0.001)$ as the preterms $(12.5 \%$ vs. $7.31 \%, p=0.075)$ and the Csection deliveries $(51.4 \%$ vs. $35.3 \%, p=0.004)$; there was no difference in 5' Apgar Score and percentage of small for gestational age (SGA).

Comparing multiple ART with spontaneous births there was a higher proportion of C-section $(92.3 \%$ vs. $70.5 \%, p=0.005)$ and SGA (35.3\% vs $17.5 \%, p=$ $0.016)$. Although no statistically significant, there were more mothers over 34 years: $26.3 \%$ vs. $14.8 \%, p=$ 0.391 and preterms $73.7 \%$ vs. $65.4 \%, p=0.600$ and were not found differences in 5' Apgar Score.

It was not possible to compare spontaneous and ART newborns regarding malformations due to the small number of this outcome.

Conclusions: We found significanty higher maternal age in singles; higher SGA newborns in multiples and higher $\mathrm{C}$-sections in both.

We didn't find significanty worse profile in the other neonatal data.

\section{Follow-up of nationwide ELBW-, ELGA -born chil- dren at 5 years in Estonia}

H. Varendia ${ }^{\mathrm{a}, \mathrm{b}}$, L. Toome ${ }^{\mathrm{a}, \mathrm{c}}$, Ü. Utsal ${ }^{\mathrm{b}}, \mathrm{K}$. Muug $^{\mathrm{b}}$, I. Marats $^{\mathrm{b}}$, M. Vasar ${ }^{\mathrm{b}}$ and P. Jüri ${ }^{\mathrm{b}}$

${ }^{a}$ Department of Paediatrics, University of Tartu, Tartu, Estonia

${ }^{\mathrm{b}}$ Children's Clinic, Tartu University Hospital, Tartu, Estonia

${ }^{\mathrm{c}}$ Tallinn Children's Hospital, Tallinn, Estonia
Estonia is a small country with $12-15000$ births annually. Perinatal mortality of ELWBI has decreased from $948 \%$ in 1992 to $370 \%$ in 2007 , whereas data about the life quality of the survivors were missing.

We performed a follow-up study of the national cohort of ELBW infants born in 2002-2003.

Methods: At five years of age 33 out of 34 survived children (13 boys, 20 girls), mean BW 850 (600-980)g; GA 26 (23-28) weeks were studied. Pediatric (incl. assessment of pulmonary function), neurological, ophthalmological, hearing examination; evaluation of psychomotor development (Movement and Kaufman ABC, Boehm \& Reynell tests) were carried out.

Results: Twelve children (35\%) were considered by all tests as healthy and normally developed; whereas 7 (20\%) of the infants were disabled ( 2 blind, 2 unable to walk independently, 3 mentally retarded). The rest of the group had moderate problems mainly in psychomotor development. We didn't find any hearing disability and serious chronic lung disease in our children.

Conclusions: About 2/3 of the ELBW infants in Estonia need continuous habilitation and developmental care at five years of age. Details of findings, correlations with perinatal factors, socioeconomic background of the families and maternal emotional status will be analyzed.

Renal agenesis and related anomalies: Preliminary results of the ultrasound multicentre study of NNSG-ISN and USPG-ISP (1990-2008)

R. Galiano, P. Cortesi, B. Baranello, R. Marzolla, G. Parisi, P. Zucchinetti, R. Agostiniani and L. Cataldi Neonatal Nephrology Study Group ISN, Ultrasound Pediatric Group-ISP, Italy

Background: In the last 40 years the antenatal and neonatal ultrasound (US) increased the early diagnosis of renal and urinary tract anomalies (UTAs), improving the wellbeing of the child and preventing some of the related problems.

Goal: To increase the knowledge about unilateral renal agenesia, and related patologies, in order to improve the quality of the care to the infant and child having nephrourological problems.

Methods: Authors performed a retrospective investigation based on US Urinary Tract neonatal screening (A period $=1990-1999$ ) and those obtained by US on newborn infants at risk for UTA (antenatal US positive exam and/or UTA in relatives) (B period $=$ 
2000-2008). This multicenter study was performed on 124.000 newborn infants enrolled in seven IInd-IIIrd level units.

The newborn infants with US suspected having unilateral agenesia were enrolled in a follow-up programme, in order to evaluate the development of a renal damage. Any associated renal and/or UTAs or other related have been evaluated.

Results: 246 children were diagnosed to have renal anomalies. 116 in the A period: 51 unilateral kidney agenesis (44\%), 57 ectopic kidney (49\%), 8 horseshoe kidneys (7\%). 128 in the B period: 48 unilateral agenesis $(37 \%), 55$ ectopic kidney (43\%), 25 horseshoe kidney (20\%).

Conclusion: The AA recommend to pay attention to all the cases of renal unilateral agenesis in order to prevent the development of renal damage, and decreasing of the health level in the next age. The continuous attention to the care is important in order to increase the level of the care to the newborn having renal or UTA, with or without other pathologies.

\section{How much does the mode of ventilation matter?}

M.T. Neto, D. Virella, P. Rodrigues, E. Leal and M. Serelha

NICU, Dona Estefânia Hospital, Centro Hospitalar de Lisboa Central, EPE, Lisbon, Portugal

Background: NICU protocol changed (2001) from intermittent mandatory (IMV) to synchronized, volume guarantee ventilation (SIPPV/PSV with VG).

Aim: To assess the effects of two ventilation modes (VM).

Methods: Effectiveness study. Inborn preterm infants, ventilated more than $12 \mathrm{~h}$; excluded if submitted to surgery, congenital malformations, weaned to $\mathrm{tC}$ PAP. Outcomes: death, bronchopulmonary dysplasia (BPD), pneumotorax, pneumonia, grade III-IV intraventricular haemorrhage (IVH), periventricular leukomalacea(PVL), ventilation days (VD), length of stay (LOS). Concurrent factors: twinning, prenatal steroids, delivery mode, gender, gestational age (GA), birth weight (BW), hyaline membrane disease (HMD), surfactant, sepsis, ductus. Models were tested by linear or binary regression.

Results: There were 194 newborns: 84 in IMV and 110 in SIMV/PSV/VG. Average GA and BW 31 weeks (23-36) and $1385 \mathrm{~g}$ (490-3450). Twenty eight twins $(14,4 \%)$, prenatal steroids $50.3 \%$, cesarean sec-

\begin{tabular}{llccc}
\hline milestone & NICU & \multicolumn{3}{c}{ gestational age (weeks) } \\
\cline { 3 - 5 } & & $24-27$ & $28-29$ & $30-31$ \\
\hline median duration of in- & Berlin & 41.5 & 34.5 & 23.0 \\
cubator therapy (d) & Innsbruck & 59.5 & 33.0 & 27.0 \\
median day of birth & Berlin & 11.0 & 12.0 & 14.0 \\
weight regain & Innsbruck & 14.0 & 12.0 & 14.0 \\
median achievement & Berlin & 83.0 & 63.0 & 41.0 \\
of full oral feeding (d) & Innsbruck & 77.5 & 49.0 & 37.0 \\
\hline
\end{tabular}

tion $75.3 \%$, HMD 70.1\%; 39 died (18\%), 10.8\% had BPD, $3.6 \%$ pneumotorax, $14.4 \%$ pneumonia. Total VT 1555 days (average $3 ; 1-80$ ) and average LOS 28 days (1-143). Death was associated to lower BW and singleton, BPD to sepsis and ductus; no association found with pneumothorax, IVH and pneumonia; PVL was associated to males, VT to ductus, pneumonia, IVH and LOS to VT, ductus and lower BW.

Conclusion: Effectiveness studies are very important to clarify the role of interventions in real settings, often hyper val orized in efficacy studies.

\section{Maturational milestones of premature infants dur- ing their NICU-career}

S. Ifflaender, J. Reichert, R.R. Wauer, W. Burkhardt and M. Rüdiger

Department of Neonatology and Pediatric Intensive Care, University Hospital Carl Gustav Carus, Dresden, Germany

Objective: Parents of preterm infants require appropriate information for informed decision making. Whereas information on morbidity and mortality are often available, data on maturational milestones of a typical "NICU-career" are often missing.

Aim: To obtain local data of maturational milestones for different gestational age groups in two different neonatal intensive care units (NICU).

Methods: Retrospective chart analysis of 240 patients of the years 2001-2005 in Berlin and Innsbruck. The following data were obtained: time of achieving full oral feeding, end of incubator therapy, time of transferring to intermediate care, day of regaining birth weight and end of respiratory support (CMV/CPAP).

Results: Important maturational milestones were achieved at different times in the two hospitals. The following table outlines some of these variations between the two NICU departments categorised by gestational age groups.

Conclusion: To inform parents on the "NICUcareer" appropriate data are required. Data, however, 
might differ between hospitals, thus local data are necessary. Differences in maturation process could reflect differences in treatment policies and could thus offer opportunities for benchmarking.

The diagnostic and prognostic value of VEGF, DR5 and ALCAM levels in newborns with posthypoxic brain damage

G.S. Golosnaya, O.Y. Popov and D.I. Algabachieva Russian State Medical University, Moscow, Russia

Background and aim: Different mechanisms of cellular injury may lead to neuronal or glial cell loss from asphyxia. Complex pathophysiological changes following asphyxia results in temporal disturbance of cell functions, inflammations and apoptosis. In this study we investigated the role of biochemical markers of apoptosis (DR5), inflammation (ALCAM), vasculal factor (VEGF) and their diagnostical significance.

Materials and methods: 80 newborns with severe hypoxic damage of CNS were examined. All methods of definition of research factors were based on principle of ELISA.The procedure was carried out according to standard protocole.

Results: DR5 level exceeded normal in all groups in first day of life. Maximum ALCAM levels were registered in all newborns during the first $48 \mathrm{~h}$ of life, later ALCAM level started to decrease. VEGF blood serum level of newborns with PVL and IVH decreased on 7 th and 28th days of life.

Conclusions: The increasing of DR5 and ALCAM level in early neonatal period and VEGF serum level decreasing after 7 th day of life is unfavorable in case of newborns with severe CNS posthypoxic damages.

Cardiac troponin I (cTnI) and N-terminal PRO-Btype natriuretic peptide (NT-proBNP) for the assessment of neonatal myocardial function

C.V. Almeida ${ }^{\mathrm{a}}$, M.R.G. Carrapato ${ }^{\mathrm{a}, \mathrm{b}}$, F. Pinto ${ }^{\mathrm{b}}$, M. Pinto $^{\mathrm{b}}$, S. Ferreira ${ }^{\mathrm{b}}$, D. Schmitt ${ }^{\mathrm{b}}$ and L. Marinho ${ }^{\mathrm{b}}$

${ }^{a}$ Health Sciences Faculty, Fernando Pessoa University, Oporto, Portugal

${ }^{\mathrm{b}}$ São Sebastião Hospital, Santa Maria da Feira, Portugal

Introduction: Fetal and neonatal myocardial lesions or dysfunction may occur secondary to intrauterine and postnatal events, leading to cell membranes disruption or cell death, with the consequent effect of intracellular substances spilling into the circulation. Little is known about these markers in the neonate and cardiac ultrasounds (US) are not always available. cTnI, NTproBNP and CK-MB may be a complementary tool for evaluation and treatment.

Objectives: 1) Determine reference ranges of biochemical markers; 2) Their correlation with ultrasounds.

Methodology: Cord and blood samples were collected from Full Term, Appropriate for Gestational Age infants of healthy mothers with uneventful pregnancies, following parental consent. NT-proBNP was determined by VIDAS NT-proBNP, CK-MB and cTnI by chemoluminescence.

Results: Median values for CK-MB declines from 79.0 to $43.0 \mathrm{U} / \mathrm{L}$ by the third day of live; cTnI rises from 0.004 to $0.066 \mathrm{ng} / \mathrm{mL}$ by 72 hours, falling to 0.0355 by 10 days; NT-proBNP peaks between 24 and 48 hours (3303.0 pg/mL), subsequently falling to $1179.5 \mathrm{pg} / \mathrm{mL}$. Between 24 to 48 hours NT-proBNP significantly rose ( $p=0.045)$ with left to right shunt as per US changes.

Discussion: CK-MB, of skeletal muscle origin in neonates, reflects delivery stress rather than myocardial injury, further demonstrated by the higher values after vacuum extraction. The rise in cTnI may be explained by a degree of myocardial involvement, albeit physiological, or due to impaired plasmatic clearance. The initial rise and subsequent fall of NT-proBNP, quite likely, represents the physiological ventricular overload of transient birth adaptation. These markers may now be used in different pathologies.

Fluid and electrolyte balance during the first week of life and risk of bronchopulmonary dysplasia in the preterm neonate

G. Rocha ${ }^{\mathrm{a}}$, O. Ribeiro ${ }^{\mathrm{b}}$ and H. Guimarães ${ }^{\mathrm{a}}$

${ }^{a}$ Division of Neonatology, Hospital de São João; Faculty of Medicine of Porto University, Porto, Portugal

${ }^{\mathrm{b}}$ Department of Biostatistics, Hospital de São João; Faculty of Medicine of Porto University, Porto, Portugal

Introduction: Fluid and electrolyte imbalances seem to be associated to an increased risk of bronchopulmonary dysplasia (BPD).

Aim: To establish an association between fluid and electrolyte balance in the first week of life and risk of BPD. 
Material and Methods: 205 neonates $<32$ weeks $\mathrm{GA} /<1250 \mathrm{~g} \mathrm{BW}$, were analysed. Longitudinal, multivariate models of generalized estimation equations (GEE) were used. A p value $<0.05$ was considered significant in all the hypothesis tests.

Results: BPD prevalence was $22 \%(n=45)$. The variation in serum values of potassium, phosphorus and creatinine along the first week of life revealed an asociation to BPD. Higher mean plasma calcium values were associated to spontaneous closure of PDA.
The use of indomethacine for PDA closure was significantly higher in BPD patients.

Conclusions: Differences in renal function and tubular handling of potassium and phosphorous are present in the first week of life among those preterm neonates that will develop BPD. The higher rate of PDA and of indomethacine use may influence these differences. Serum levels of calcium seem to play a role in spontaneous ductus closure. 